\title{
Health related quality of life in Parkinson's disease: a prospective longitudinal study
}

\author{
Karen H Karlsen, Elise Tandberg, Dag Årsland, Jan P Larsen
}

\begin{abstract}
Objectives-To examine the change over time in health related quality of life (HRQL) in a community based cohort of patients with Parkinson's disease.

Methods-One hundred and eleven patients were evaluated for HRQL in 1993 and then again in a follow up study 4 years later. The patients included in the study in 1993 were derived from a prevalence study of patients with Parkinson's disease in the county of Rogaland, Norway. The HRQL was measured by the Nottingham health profile (NHP). At both evaluations clinical and demographic variables were determined during semistructured interviews and by clinical examinations by a neurologist.
\end{abstract}

Results-During the 4 year follow up period there was a significant increase in NHP scores, reflecting a decreased HRQL, in the dimensions of physical mobility, emotional reactions, pain, and social isolation. In the same time period mean total NHP score increased from 120.0 (SD 102.6) to 176.0 (SD 119.4) $(p<0.01)$. There were no clinical or demographic factors found in 1993 that identified patients at higher risk for developing decreased HRQL. Increased UPDRS score (unified Parkinson's disease rating scale) and Hoehn and Yahr stage during the 4 year study period correlated with increased NHP scores. Even though there was no increase in depressive symptoms or self reported insomnia, these symptoms, together with lower Schwab and England score, were the most important factors for a poor HRQL in 1997.

Conclusions-Parkinson's disease has a substantial impact on HRQL. Despite modern care, we found a significantly increased distress during the 4 year follow up period. Increased parkinsonism, measured by UPDRS and Hoehn and Yahr stage, correlated with increased stress, not only in the dimension of physical mobility, but also in the areas of pain, social isolation, and emotional reactions. In addition to the clinical examination, HRQL scoring provides valuable information on the total health burden of Parkinson's disease in both cross sectional and longitudinal evaluations, and contributes to a more comprehensive picture of the total disease impact.

(F Neurol Neurosurg Psychiatry 2000;69:584-589)

Keywords: Parkinson's disease; health related quality of life; Nottingham health profile; longitudinal study
Parkinson's disease is a common neurodegenerative disorder, affecting $100-150$ patients per 100000 population. ${ }^{1-3}$ The main focus in evaluation and treatment of patients with Parkinson's disease has been on the motor disability of the disease. During the past decade, however, greater emphasis has been placed on the total impact of Parkinson's disease on the patient's life, thus taking into consideration not only the physical impairment of the disease, but also various non-motor symptoms including depression, cognitive impairment, and sleep disturbances. ${ }^{4-7}$ These symptoms intensify as the disease progresses, and result in a considerable burden of illness associated with Parkinson's disease. As the medical interventions aim to produce symptomatic improvement, the concept of health related quality of life (HRQL) should be implicit in outcome based investigation and data about the HRQL, from the patients' perspective, are fundamental to understanding the effectiveness of care.

Few studies have to date been published describing the impact of Parkinson's disease on patients' HRQL. This approach to evaluation of the patients' situation has shown that the disease has a considerable negative consequence, not only on physical mobility, but also on mood, social functioning, sleep, and fatigue. ${ }^{8-12}$ Some studies have also to different degrees explored which clinical factors are the most important for increased distress in patients with Parkinson's disease. ${ }^{13}$ This information is important in the development of optimal management strategies for the best possible wellbeing among patients with Parkinson's disease and in the identification of potentially modifiable factors associated with adverse outcomes in this frail population.

We have earlier reported the results from a community based study of HRQL in patients with Parkinson's disease, using the Nottingham health profile. ${ }^{813}$ As in previous studies, we found in this unselected patient population that Parkinson's disease had a negative impact on all the measured dimensions of quality of life. The most important clinical factors associated with a poor quality of life were the presence of depressive symptoms and insomnia.

Although quality of life in patients with Parkinson's disease has been increasingly explored and described, there is no information on the longitudinal evolution of distress and wellbeing in patients with Parkinson's disease. Such information could indicate which patients need a closer follow up in future management and provides insight into the change over time of HRQL in a group of patients with a traditional motor control focused treatment. To consider 
these issues we have re-examined the HRQL among patients from a community based 1993 prevalence population with Parkinson's disease in a 4 year follow up study.

\section{Materials and methods}

PATIENTS

One hundred and eleven patients with idiopathic Parkinson's disease could be assessed for HRQL in both 1993 and 4 years later. The total patient material in 1993 comprised 245 patients diagnosed with Parkinson's disease in the county of Rogaland, in an epidemiological study on 1 January $1993 .^{3}$ The prevalence rate was 110.9/100 000 inhabitants. Complete case ascertainment in the 1993 prevalence study was attempted through an extensive search for patients known to the specialist neurological service, the general practitioners in the area, nursing homes, district nurses, and home health workers. The diagnosis was made according to explicit diagnostic criteria. ${ }^{14}$ Two hundred and thirty three of these patients were included in the first evaluation of quality of life. Three patients died between prevalence day and scheduled examination. Two patients did not wish to participate and seven patients could not be evaluated due to severe dementia. At the second evaluation in 1997, 111 patients were available for evaluation of HRQL. Eighty seven had died, one patient had emigrated, 18 could not be evaluated because of severe dementia, 11 did not chose to participate, and seven of the 245 were rediagnosed as not having Parkinson's disease. The study was approved by the regional ethics committee at the University of Bergen.

STUDY DESIGN AND EVALUATION PROGRAMME The study was a prospective longitudinal study of change in HRQL in a community based cohort of patients with Parkinson's disease. The patients were examined in 1993 and in 1997 and the study was part of a larger evaluation of non-motor symptoms in patients with Parkinson's disease. All patients were interviewed and evaluated by a neurologist. The quality of life questionnaire was self administered. The patients with problems filling in the forms were allowed assistance from family members or from the nurse at the outpatient clinic. We used semistructured interviews to obtain information on disease history, demographic variables, and medication. The patients were evaluated with the unified Parkinson's disease rating scale (UPDRS), ${ }^{15}$ including the Hoehn and Yahr staging ${ }^{16}$ and the Schwab and England scale. ${ }^{17}$ The UPDRS can be divided into four subscales: mentation, behaviour, and mood (UPDRS mental score); activities of daily living (UPDRS ADL score); motor examination (UPDRS motor score); and complications of therapy. The first three subscales are quantitative five point scales ( $0-4$ score), the fourth is a yes/no scale. The modified Hohn and Yahr scale consists of seven categories ranging from 0.0-5.0. For analytical purposes we grouped 1.0 and 1.5 as one category (unilateral disease) and 2.0 and 2.5 as mild to moderate bilateral disease. The Schwab and
England scale is scored from $0-100$ in 10 point increments, with 0 describing a vegetative state and 100 a completely independent healthy state. We recorded the presence or absence of wearing off fluctuations (UPDRS item 36), dyskinesia (UPDRS item 32, where a score of more than 0 was recorded "dyskinesia present"), and psychotic symptoms (UPDRS item 2 , where a score of 2 or more was recorded as "psychotic symptoms present"). We also evaluated cognitive function (mini mental state examination (MMSE) $)^{18}$ ) and depressive symptoms (Beck depression inventory $(\mathrm{BDI})^{19}$ ). The presence or absence of self reported insomnia was recorded. The neurologist performing the clinical examination in 1997 was blinded to the results from the 1993 assessments.

HRQL MEASUREMENTS

We employed the Nottingham health profile (NHP, part 1). ${ }^{20-22}$ The NHP was developed during the 1970s in the United Kingdom as a measure of perceived health. Part 1 consists of 38 items (yes/no), covering the domains of emotional reactions (nine items), energy (three items), pain (eight items), physical mobility (eight items), sleep (five items), and social isolation (five items). Each item carries a specific weight, so that within each dimension the weighted scores range from 0 (indicating good health) to 100 (indicating poor health). The NHP has undergone extensive evaluation of psychometric properties and has been validated in several patient populations. ${ }^{22-24}$ There is, however, no generally accepted norm as to what constitutes a clinically relevant decrease in quality of life (reflected by higher scores on the NHP). A 10 point increase in score will most likely affect patients differently if the primary measured score was 0 (no reported problems at all) or 50 (indicating that the disease already has a high negative impact on quality of life). We therefore chose to define a significant worsening of HRQL as a more than 30\% increase in distress score. By this we could divide the patient population into two groups, those with a significant deterioration of quality of life during the 4 year follow up period and those patients without significantly higher distress scores.

\section{STATISTICAL METHODS}

All data analyses were performed using the software program SPSS 7.5 (SPSS Inc; Chicago). For analyses of rates and proportions we used $\chi^{2}$ (Fisher's exact test when an expected cell frequency was $<$ five). The data were not normally distributed and non-parametric tests of significance were used. For comparisons of continuous data we used the Wilcoxon signed ranks test (paired data) and Mann-Whitney test (two unrelated samples). For tests of correlation we used the Spearman correlation coefficient. We performed multiple regression analysis with the NHP total score as the dependent variable and logistic regression analysis with the proportion of patients with a more than $30 \%$ increase in NHP total score as the outcome variable. In that no formal corrections were made for multiple comparisons, we 
Table 1 Demographic and clinical data for 111 patients with Parkinson's disease

\begin{tabular}{|c|c|c|c|c|c|}
\hline & 1993 & 1997 & $p$ Value $^{\star}$ & $\begin{array}{l}\text { Mean change } \\
(S D)\end{array}$ & $95 \%$ CIt \\
\hline Age $(y)($ mean $(S D))$ & $69.8(8.0)$ & & & & \\
\hline No of women $(\%)$ & $63(56.8)$ & & & & \\
\hline Duration of disease (y) (mean (SD)) & $8.5(5.0)$ & & & & \\
\hline Duration of medication (y) (mean (SD)) & $6.0(4.5)$ & & & & \\
\hline \multicolumn{6}{|l|}{ UPDRS score (mean (SD)) } \\
\hline Mental & $2.6(1.9)$ & $3.8(2.8)$ & 0.000 & $1.2(2.7)$ & $(0.7$ to 1.7$)$ \\
\hline $\mathrm{ADL}$ & $10.3(6.7)$ & $16.8(9.6)$ & 0.000 & $6.5(7.1)$ & (5.1 to 7.8 ) \\
\hline Motor & $21.2(12.4)$ & $29.2(19.1)$ & 0.000 & $7.9(13.6)$ & (5.3 to 10.5$)$ \\
\hline Complications & $2.9(3.6)$ & $3.5(3.3)$ & 0.028 & $0.5(3.0)$ & $(-0.1$ to 1.1$)$ \\
\hline Total & $36.9(20.8)$ & $52.6(29.4)$ & 0.000 & $15.7(19.7)$ & (12.0 to 19.5$)$ \\
\hline \multicolumn{6}{|l|}{ Hoehn and Yahr stage (n (\%)) } \\
\hline 1.0 and 1.5 (unilateral), & $28(25.2)$ & $9(8.1)$ & & & \\
\hline 2.0 and 2.5 (moderate) & $51(45.9)$ & $38(34.2)$ & & & \\
\hline 3.0 (moderate to advanced) & $20(18.0)$ & $29(26.6)$ & & & \\
\hline 4.0 (advanced) & $11(9.9)$ & $19(17.4)$ & & & \\
\hline 5.0 (advanced) & $1(0.9)$ & $14(12.8)$ & & & \\
\hline Mean score (SD) & $2.3(0.9)$ & $3.1(1.0)$ & 0.000 & $0.7(0.8)$ & $(0.6$ to 0.9$)$ \\
\hline Levodopa dose (mg/day) (mean (SD)) & $512.1(247.9)$ & $649.3(367.5)$ & 0.000 & $137.1(279.0)$ & (83.7 to 190.6$)$ \\
\hline BDI score (mean (SD)) & $11.6(6.9)$ & $12.2(7.2)$ & 0.369 & $0.6(8.0)$ & $(-1.0$ to 2.2$)$ \\
\hline MMSE score (mean (SD)) & $27.5(3.4)$ & $26.2(5.3)$ & 0.014 & $-1.3(4.6)$ & $(-2.2$ to -0.4$)$ \\
\hline Self reported insomnia (n (\%)) & $69(62.2)$ & $57(51.4)$ & 0.000 & & \\
\hline Wearing off fluctuations (n (\%)) & $27(24.3)$ & $42(37.8)$ & 0.000 & & \\
\hline Presence of dyskinesia $(\mathrm{n}(\%))$ & $31(27.9)$ & $41(36.9)$ & 0.000 & & \\
\hline
\end{tabular}

*Wilcoxon; †confidence interval of the difference.

chose a conservative level of significance, and only $\mathrm{p}$ values $<0.01$ were considered statistically significant throughout the study. All p values are two tailed.

\section{Results}

In table 1 the demographic and clinical characteristics of the 111 patients in 1993 and 4 years later are presented. The mean age at the first evaluation was 69.8 years, and the patients were equally divided between men and women. Mean duration of disease was 8.5 years, and mean duration of dopaminergic drug therapy was 6.0 years. Seventy nine patients had Hoehn and Yahr stage 2.5 or less at the first evaluation. Four years later only 47 patients were still classified as having only mild to moderate disease. Mean total UPDRS score increased from 36.9 (SD 20.8) in 1993 to 52.6 (SD 29.4) in 1997. The proportion of patients with wearing off fluctuations rose from $24.3 \%$ to $37.8 \%$ and the proportion of patients with dyskinesia increased by $10 \%$. Daily dose of levodopa increased from a mean of $512.1 \mathrm{mg} /$ day to $649.3 \mathrm{mg} /$ day. There was no increase in depressive symptoms measured by BDI, but we did see a small deterioration of cognitive function, measured by the MMSE. The percentage of patients with self reported insomnia declined from $62.2 \%$ to $51.4 \%$.

The NHP scores in 1993 and 1997 are presented in table 2 . The mean total NHP distress score increased by a mean of 56 points, from
120.0 (SD 102.6) to 176.0 (SD 119.4). For almost $60 \%$ of the patients the total increase in NHP score was more than $30 \%$ compared with the score at the primary evaluation in 1993. The dimensions of the NHP with the highest deterioration during the 4 year period were physical mobility, emotional reactions, pain, and social isolation. There was no mean change in distress score in the dimension measuring sleep problems; despite this, more than $30 \%$ of the patients experienced a greater than $30 \%$ increase in score. Many patients did, however, experience an improvement in this dimension. There was an increase in score in the dimension measuring problems with low energy; this was, however, not statistically significant at the $1 \%$ level. At both the primary evaluation in 1993 and the second evaluation in 1997 the dimensions of the NHP that were most affected were physical mobility, sleep, energy, and pain.

We did not find any association between any of the following demographic and clinical variables registered in 1993 and the increase in NHP score after 4 years: age, sex, duration of dopaminergic therapy, mean levodopa dose, depressive symptoms (BDI score), cognitive impairment (MMSE score), total UPDRS score, or Hoehn and Yahr stage. Table 3 shows the results for total NHP score. The same analyses for all the six subdimensions of the NHP gave non-significant results. In addition, we performed a logistic regression analysis with

Table 2 Nottingham health profile (NHP) scores* in 111 patients with Parkinson's disease

\begin{tabular}{|c|c|c|c|c|c|c|}
\hline & $\begin{array}{l}1993 \\
\text { Mean (SD) }\end{array}$ & $\begin{array}{l}1997 \\
\text { Mean (SD) }\end{array}$ & $p$ Valuet & $\begin{array}{l}\text { Proportion with } \\
\text { more than } 30 \% \\
\text { increase in score }\end{array}$ & Mean change (SD) & $95 \% C I \ddagger$ \\
\hline Emotional reactions & $10.7(15.8)$ & $18.7(24.5)$ & 0.000 & 39.6 & $8.1(22.9)$ & (3.7 to 12.4 ) \\
\hline Energy & $20.5(27.5)$ & $28.1(33.3)$ & 0.039 & 30.6 & $7.5(36.0)$ & (0.8 to 14.3$)$ \\
\hline Pain & $19.2(24.9)$ & $29.6(26.5)$ & 0.000 & 52.3 & $10.4(23.7)$ & (6.0 to 14.9$)$ \\
\hline Physical mobility & $26.7(27.2)$ & $44.3(29.5)$ & 0.000 & 56.8 & $17.5(28.3)$ & (12.2 to 22.9$)$ \\
\hline Sleep & $29.5(31.4)$ & $30.0(30.5)$ & 0.850 & 33.3 & $0.5(25.7)$ & $(-4.3$ to 5.3$)$ \\
\hline Social isolation & $13.4(19.7)$ & $25.4(26.0)$ & 0.000 & 47.7 & $11.9(27.1)$ & ( 6.8 to 17.0$)$ \\
\hline Total NHP & $120.0(102.6)$ & $176.0(119.4)$ & 0.000 & 58.6 & $56.0(110.8)$ & (35.1 to 76.8 ) \\
\hline
\end{tabular}

$\star$ Range of possible scores from 0 to 100: 0, best possible health status; 100, worst possible health status; †Wilcoxon signed ranks test; ‡confidence interval of the difference. 
Table 3 Deterioration rate. Proportion of patients with Parkinson's disease with more than $30 \%$ increase in the total NHP score during the 4 year follow up period

\begin{tabular}{|c|c|c|c|}
\hline Patient characteristics in 1993 & Deterioration rate & $\%$ & $p$ Value * \\
\hline \multicolumn{4}{|l|}{ Age (y): } \\
\hline$<65$ & $8 / 17$ & 47.1 & \multirow{3}{*}{0.276} \\
\hline $65-75$ & $38 / 67$ & 56.7 & \\
\hline$>75$ & $19 / 27$ & 70.4 & \\
\hline \multicolumn{4}{|l|}{ Sex: } \\
\hline Male & $25 / 48$ & 52.1 & \multirow[b]{2}{*}{0.227} \\
\hline Female & $40 / 63$ & 63.5 & \\
\hline \multicolumn{4}{|l|}{ UPDRS total score: } \\
\hline$<20$ & $9 / 20$ & 45.0 & \multirow{4}{*}{0.023} \\
\hline $20-39$ & $33 / 50$ & 66.0 & \\
\hline $40-59$ & $20 / 29$ & 69.0 & \\
\hline$>60$ & $3 / 12$ & 25.0 & \\
\hline \multicolumn{4}{|l|}{ Hoehn and Yahr stage: } \\
\hline 1 and 1.5 & $16 / 28$ & 57.1 & \multirow{4}{*}{0.275} \\
\hline 2 and 2.5 & $32 / 51$ & 62.7 & \\
\hline 3 & $13 / 20$ & 65.0 & \\
\hline 4 and 5 & $4 / 12$ & 33.3 & \\
\hline \multicolumn{4}{|c|}{ Duration of dopaminergic treatment $(\mathrm{y})$ : } \\
\hline$<3$ & $22 / 32$ & 68.8 & \multirow{3}{*}{0.338} \\
\hline $3-10$ & $32 / 57$ & 56.1 & \\
\hline$>10$ & $11 / 22$ & 50.0 & \\
\hline \multicolumn{4}{|l|}{ Levodopa dose (mg/day): } \\
\hline$<400$ & $14 / 27$ & 51.9 & \multirow{3}{*}{0.391} \\
\hline $400-700$ & $39 / 61$ & 63.9 & \\
\hline$>700$ & $11 / 22$ & 50.0 & \\
\hline \multicolumn{4}{|l|}{ MMSE score: } \\
\hline$<24$ & $4 / 12$ & 33.3 & \multirow[b]{2}{*}{0.071} \\
\hline$\geqslant 24$ & $61 / 99$ & 61.6 & \\
\hline \multicolumn{4}{|l|}{ BDI score: } \\
\hline$<18$ & $53 / 87$ & 60.9 & \\
\hline$\geqslant 18$ & $11 / 21$ & 52.4 & 0.475 \\
\hline
\end{tabular}

$\star$ Pearson $\chi^{2}$; Fisher's exact test when expected cell count $<5$.

Table 4 Correlation * between increase in NHP scores and increase in total UPDRS scores and between increase in NHP score and a higher Hoehn and Yahr stage

\begin{tabular}{lllll}
\hline & $\begin{array}{l}\text { Increase in total } \\
\text { UPDRS score }\end{array}$ & p Value & $\begin{array}{l}\text { Increase in Hoehn } \\
\text { and Yahr stage }\end{array}$ & p Value \\
\hline Emotional reactions & 0.267 & 0.005 & 0.162 & 0.092 \\
Energy & 0.269 & 0.005 & 0.280 & 0.003 \\
Pain & 0.255 & 0.008 & 0.274 & 0.004 \\
Physical mobility & 0.356 & 0.000 & 0.385 & 0.000 \\
Sleep & 0.123 & 0.204 & 0.278 & 0.003 \\
Social isolation & 0.277 & 0.004 & 0.304 & 0.001 \\
Total NHP & 0.370 & 0.000 & 0.434 & 0.000 \\
\hline
\end{tabular}

${ }^{\star}$ Spearman correlation coefficient.

the proportion of patients with a more or less than $30 \%$ increase in NHP score as the outcome variable in the model and the following variables from the 1993 data as possible explanatory variables: age, sex, duration of dopaminergic treatment, mean levodopa dose, depressive symptoms (BDI score), cognitive impairment (MMSE score), total UPDRS score, and Hoehn and Yahr stage. The model did not yield any significant results. We could therefore not identify any factors that in advance could predict which patients had a higher risk of developing decreased HRQL, measured by increased NHP score.

There was a clear association between increased parkinsonism, both measured by total UPDRS score and Hoehn and Yahr stage, and increased NHP score during the follow up period. For total UPDRS score, all the dimensions of the NHP except sleep showed significant correlations. The only dimension that did not correlate with a higher Hoehn and Yahr stage was emotional reactions (table 4).

We used multiple regression analysis to explore the association between clinical and demographic variables in 1997 and NHP score. We entered NHP total score (1997) as the dependent variable and as possible influential factors we entered the following variables: age, sex, BDI score, presence of self reported insomnia, Hoehn and Yahr stage, UPDRS subscores (mental score, ADL score, motor score, and complications of therapy), Schwab and England score, presence of wearing off fluctuations, presence of dyskinesia, daily levodopa dose, and duration of levodopa medication (all data from the 1997 evaluation). The model explained $47 \%$ of the variation in NHP score. The variables that contributed to the model $(\mathrm{p}<0.01)$ were BDI score $(B=6.7, \mathrm{SE}=1.2$, $t=5.7)$, presence of self reported insomnia $(B=56.5, \mathrm{SE}=16.8, t=3.7)$, and Schwab and England score $(B=-1.6, \mathrm{SE}=0.5, t=-3.2)$. Finally, we performed multiple regression analysis with NHP total score in 1997 as the outcome variable and the same demographic and clinical data as mentioned above, but from the 1993 study, as possible explanatory factors. In addition, the total NHP score from 1993 was included. The total model explained 39\% of the variation in total NHP (1997) score, but with only NHP (1993) $(B=0.4, \mathrm{SE}=0.1, t=4.0)$ and daily levodopa dose $(B=0.1, \mathrm{SE}=0.0$, $t=3,4$ ) remaining in the model at the $1 \%$ chosen level of significance.

\section{Discussion}

This study is the first to describe the longitudinal evolution of HRQL over time in patients with Parkinson's disease. We found that the patients reported a significant deterioration in several domains, not only decreased physical mobility, but also an increase in distress in the areas of pain, social isolation, and emotional reactions. The traditional rating scales do not cover these dimensions for Parkinson's disease. Our findings emphasise that Parkinson's disease affects patients' lives in a broader sense than merely by physical impairment, and that despite modern treatment, the impact of the disease increases as the disease progresses.

The study was part of a prevalence study of Parkinson's disease and extensive methods were employed to obtain total case ascertainment. ${ }^{3}$ There will, however, always be patients who have not yet come to the attention of the medical healthcare system, and our 1993 prevalence figure will therefore be lower than the true figure. The medical system in the area is, however, well developed, easily accessed, and independent of economic or social status, and we think most cases of Parkinson's disease were included in the study. We used explicit criteria and excluded patients with a low probability for idiopathic Parkinson's disease. ${ }^{14}$ Of the 233 patients that were available for evaluation of HRQL in 1993, 111 were still able to provide complete data sets 4 years later. Most of the patients lost to follow up were due to patient death; however, some bias may have entered by virtue of 11 patients refusing to participate and seven patients not evaluated due to severe dementia.

The assessment of HRQL, defined as self perceived health status, is increasingly regarded as a distinct outcome measure in patients with many chronic diseases. ${ }^{24} 25$ It adds the patient perspective to the physicians' evaluation and enables the assessment of disease conse- 
quences on an individual level. Parkinson's disease is a chronic disease that has consequences not only on patients' functional abilities but also on mental, emotional, and social health domains. When assessing the overall effectiveness of new management strategies, HRQL is an important outcome measure. Information on HRQL is also used in the decision making process on the allocation of resources in health care at the national health policy level. ${ }^{25}$

The NHP measures aspects of quality of life that we think are relevant to patients with Parkinson's disease. It has undergone rigorous testing for reliability and validity and is easily administered and understood. ${ }^{20}{ }^{21}$ A limitation of the NHP is that it shows the highest sensitivity in the upper score range and that changes of HRQL in patients with a lesser health burden may not be detected. The changes expected are often small when compared with other disease states. The present study does, however, show that the NHP is sensitive to change over time in patients with Parkinson's disease. Because there is no generally accepted norm as to what constitutes a clinically relevant decrease in quality of life, measured by the NHP, we chose to define a more than $30 \%$ increase in distress score between the two evaluation points as a cut off value.

The NHP is a generic instrument for evaluation of HRQL. It has, therefore, not been developed as a questionnaire that should detect the specific problems that may be experienced by patients living with Parkinson's disease. In recent years disease specific instruments have been introduced, including the Parkinson's disease questionnaire 39 (PDQ-39) ${ }^{26}$ and the Parkinson's disease quality of life questionnaire (PDQL). ${ }^{27}$ The PDQ-39 has been shown to be a reliable and valid instrument for patients with Parkinson's disease ${ }^{26} 27$ and is now in general use when examining HRQL in the disease. This instrument was, however, not available at the time the present study was planned. The results from this and previous studies ${ }^{813}$ show, however, that the NHP also gives meaningful and valuable information on HRQL in Parkinson's disease.

As expected from the increase in UPDRS score and shift towards higher Hoehn and Yahr stages during the follow up period we found that there was an increased score in the NHP dimension of physical impairment. There is high agreement between the physicians' evaluation of deterioration of motor function and the patients' self evaluation of physical mobility. Although there was no increase in depressive symptoms measured by the BDI, there was a significantly higher distress score in the dimension of the NHP measuring emotional reactions. This could be because the dimension of emotional reactions in the NHP measures a wider range of emotional problems, not only depressive symptoms. It is also possible that NHP may be more sensitive to change than $\mathrm{BDI}$ in this population group. Another possible explanation for the lack of progression of depressive symptoms could be that a more aggressive treatment of depression followed the
1993 study. We did not see an increase in sleep problems in the 4 year follow up period, and the increase in score in the dimension of low energy was not statistically significant. This indicates that these problems occur early in the disease, and do not increase as the disease progresses. These findings do, however, need to be further investigated.

We did not find any association between any demographic or clinical findings in 1993 and reduced HRQL during the follow up period. We could therefore not identify specific patient groups that were at special risk for experiencing an increased health burden in the future. There was, however, a clear association between progression of the disease measured by the Parkinson's disease rating scales (UPDRS scores and Hoehn and Yahr stage), and an increased NHP score. This association was not only apparent in the dimension of physical mobility, but also in all the other measured dimensions of HRQL. This is important to bear in mind when evaluating the full impact of the progression of the disease on the patient. This awareness enhances the possibility of identifying, and when possible treating, symptoms that may not be apparent from the standard evaluation of parkinsonism, such as increased pain and emotional problems.

We have earlier shown that presence of depressive symptoms and insomnia are important factors for HRQL in patients with Parkinson's disease. ${ }^{13}$ In the present study we did not see an increase in these symptoms during the 4 year follow up period. The higher distress scores in the NHP could thus not be explained by a deterioration of depression and sleep disturbances. The lack of measurable progression in depressive symptoms is in accord with earlier studies evaluating the longitudinal change in depression in patients with Parkinson's disease.$^{28}$ However, when performing multiple regression analysis, we found that the patients in 1997 still reported that the presence of depressive symptoms, insomnia, and a lower score on the Schwab and England scale were the most important predictors for a high distress score on the NHP scale.

There are several possible limitations in evaluating these results. Social factors, including loss of spouse or other relatives, changes in living situation, or degree of social contact may also be expected to influence the observed variation in HRQL. We did not evaluate the presence of concurrent diseases, which would be expected to be present in this age group, or the effects of medications taken for diseases other than Parkinson's disease. The emergence of new diseases or the progression of concomitant illness would be expected to account for some of the worsening of scores. The aim of the study was, however, to describe a "true to life" situation in a non-selected patient population, and controlling for other diseases would disturb this perspective.

This study shows that Parkinson's disease has a substantial impact on HRQL and that despite treatment there is a significant deterioration over time. The health burden of Parkinson's disease can be assessed on several levels, 
each of which has a different perspective. Symptom scales, generic HRQL measures, and disease specific measures can all be used for this, either alone or in combination. HRQL scoring cannot replace traditional symptom scoring such as the UPDRS. It does, however, provide valuable information on the total health burden of Parkinson's disease, in both cross sectional and longitudinal evaluations, and contributes to a comprehensive picture of the total disease impact.

We thank Assistant Professor Kjell Jørgensen, Norwegian School of Management, for his advice with the statistical analyses and the Inge Stensland Foundation for financial support for the study.

1 Martilla RJ, Rinne UK. Epidemiology of Parkinson's disease in Finland. Neurol Scand 1976;53:81-102.

2 Mutch WJ, Dingwall-Fordyce I, Downie AW, et al. Parkinson's disease in a Scottish city. BMF 1986;292:5346.

3 Tandberg E, Larsen JP, Nessler EG, et al. The epidemiology of Parkinson's disease in the county of Rogaland, Norway. Mov Disord 1995;10:541-9.

4 Cummings JL. Depression and Parkinsons' disease: a review. Am f Psychiatry 1992;149:443-54.

5 Tandberg E, Larsen JP, Aarsland D, et al. The occurrence of depression in Parkinson's disease. A community-based study. Arch Neurol 1996;53:175-79.

6 Årsland D, Tandberg E, Larsen JP, et al. Frequency of dementia in Parkinson's disease. Arch Neurol 1996;53:53842 .

7 Lees AJ, Blackburn NA, Campell VL. The nighttime problems of Parkinson's disease. Clin Neuropharmacol 1988;6. 512-19.

8 Karlsen KH, Larsen JP, Tandberg E, et al. Quality of life measurements in patients with Parkinson's disease: a community-based study. Eur F Neurol 1998;5:443-50.

9 Friedman J, Friedman H. Fatigue in Parkinson's disease. Neurology 1993;43:2016-18.

10 Chrischilles EA, Rubenstein LM, Voelker MD, et al. The health burdens of Parkinson's disease. Mov Disord 1998;13 406-13.

11 de Boer AGEM, Wijker W, Speelman JD, et al. Quality of life in patients with Parkinson's disease: development of a questionnaire. I Neurol Neurosurg Psychiatry 1996;61:70-4.
12 Longstreth WT Jr, Nelson L, Linde M, et al. Utility of the sickness impact profile in Parkinson' disease. 7 Geriatr Psysickness impact profile in Park
chiatry Neurol 1992;5:142-8.

13 Karlsen KH, Larsen JP, Tandberg E, et al. The influence of clinical and demographic variables on quality of life in Parkinson's disease. $\mathcal{F}$ Neurol Neurosurg Psychiatry 1999;66: 431-5.

14 Larsen JP, Dupont E, Tandberg E. The clinical diagnosis of Parkinson's disease: proposal of diagnostic subgroups classified at different levels of confidence. Acta Neurol Scand 1994;84:242-51.

15 Fahn S, Elton RL. Members of the UPDRS Development Committee: unified Parkinson's disease rating scale. In: Fahn S, Marsden CD, Calne DB, et al, eds. Recent developments in Parkinson's disease. Florham Park: MacMillan Health Care Information, 1987:153-63.

16 Hoehn M, Yahr M. Parkinsonism: onset, progression and mortality. Neurology 1967;17:427-42.

7 Schwab RS, England AC. Projection technique for evaluating surgery in Parkinson's disease. In: Gillingham FJ, Donaldson IML, eds. Third Symposium on Parkinson's disease. Edinburgh: Livingstone, 1969.

18 Folstein MF, Folstein SE, McHugh PR. Mini-mental state: a practical method for grading the mental state of patients for the clinicians. $\mathcal{F}$ Psychiatr Res 1975;12:189-98.

19 Beck AT, Ward CH, Mendelson M, et al. An inventory for measuring depression. Arch Gen Psychiatry. 1961;4:56171 .

20 Hunt SM, McEwen J, McKenna SP. Measuring health status: a new tool for clinicians and epidemiologists. $\mathcal{F}$ Coll Gen Pract 1985;35:185-8.

21 Hunt SM, McEwen J, McKenna SP. Measuring health status. London: Croom Helm, 1986

22 Jenkinson C, Fitzpatrick R, Argyle M. The Nottingham health profile: an analysis of its sensitivity in differentiating illness groups. Soc Sci Med 1988;27:1411-14.

23 Essink-Bot ML, van Royen L, Krabbe P, et al. The impact of migraine on health status. Headache 1995;35:200-6.

24 Dornan TL, Peck GM, Dow JDC, et al. A community survey of diabetes in the elderly. Diabet Med 1992;9:860-5.

25 TenVergert EM, Essink-Bot ML, Geertsma A, et al. The effect of lung transplantation on health-related quality of effect of lung transplantation
life. Chest 1998;113:358-64.

26 Peto V, Jenkinson C, Fitzpatrick R, et al. The development and validation of a short measure of functioning and well being for individuals with Parkinson's disease. Qual Life Res 1995;4:241-8

27 Hobson P, Holden A, Meara J. Measuring the impact of Parkinson's disease with the Parkinson's disease quality of life questionnaire. Age Ageing 1999;28:341-6.

28 Brown RG, MacCarthy B, Gotham AM, et al. Depression and disability in Parkinson's disease: a follow up of 132 cases. Psychol Med 1998;18:49-55. 